\title{
High Dose Methylprednisolone: Effective in Pregnancy Associated Post-splenectomy Refractory Chronic Immune Thrombocytopenic Purpura
}

\author{
Singh A $\mathbf{A}^{1}$, Solanki $\mathbf{A}^{2}$ \\ ${ }^{1}$ Department of Transfusion Medicine and Immunohematology, The Mission Hospital, Durgapur, West Bengal, India. ${ }^{2}$ Department of \\ Transfusion Medicine, Sanjay Gandhi Post Graduate Institute of Medical Sciences, Lucknow, Uttar Pradesh, India.
}

\begin{abstract}
We present a case of 23 years multigravid woman $\left(\mathrm{G}_{2} \mathrm{P}_{0+1}\right)$ with chronic idiopathic thrombocytopenic purpura (ITP), refractory to treatment in forms of oral steroid, dapsone, azathioprine and splenectomy. She presented to the hospital in third trimester of pregnancy, with reduced platelet counts and purpuric rashes over abdomen and both upper extremeties. There was a past history of spontaneous abortion at 18 weeks. The patient responded to intravenous high dose methylprednisolone (HDMP) infusion leading to delivery of full term male baby. The baby also required treatment for thrombocytopenia with intravenous immunoglobulin (IVIG). This case lays down the importance of HDMP in form of using it effectively in managing acute crisis of low platelet counts in pregnant patients with ITP refractory to splenectomy.
\end{abstract}

Keywords: chronic idiopathic thrombocytopenic purpura, intravenous immunoglobulin, methylprednisolone, splenectomy.

\section{INTRODUCTION}

Idiopathic thrombocytopenic purpura with an overall incidence of 1.6 per 100,000 per year is particularly more common in women of child bearing age group. ${ }^{1,2}$ In pregnant women ITP is the most common cause of thrombocytopenia in first and second trimester of pregnancy affecting 1 to 2 women of every 10,000 pregnancies. ${ }^{3}$ ITP is an autoimmune disorder, where platelets are destroyed due to binding of platelet autoantibodies to the antigen on platelet surface. These platelet autoantibodies can also cross placenta and are responsible for thrombocytopenia in some infants. ${ }^{4}$ Chronic ITP refractory to splenectomy in obstetrics poses a life threatening hemostatic risk during delivery hence managing such type of patient is a real challenge. We report here a case of management of acute crisis of low platelets in a pregnant female of chronic ITP refractory to splenectomy.

\section{CORRESPONDENCE}

Dr Abhay Singh

Department of Transfusion Medicine and Immunohematology

The Mission Hospital, Durgapur-713212, West Bengal, India

Email: abhaysgpgi@gmail.com

Phone: +91-9454307273

\section{CASE}

A 23 years multigravid patient $\left(\mathrm{G}_{2} \mathrm{P}_{0+1}\right)$ in her third trimester (at 33 weeks gestation) of pregnancy, weight $62 \mathrm{~kg}$ and blood group $\mathrm{O} \mathrm{Rh}(\mathrm{D})$ positive, presented to our hospital with chief complaints of purpuric rashes over anterior abdomen and both upper extremities with epistaxis since last one week. Physical examination revealed no icterus or lymphadenopathy. She had purpuric rashes over abdomen and both upper limbs with no hepatomegaly. Cardiovascular, chest and neurological examination yielded no significant findings. The patient had a past history ITP since last eight years and was maintained on oral medications in form of steroid, folic acid and azathioprine. Past laboratory reports yielded bone marrow to be normocellullar with increased megakaryocytes. Kidney and liver function tests were within normal limits. Antinuclear antibody (ANA) was negative and double stranded DNA (DS DNA) was within normal range. Being non-responsive to oral medications patient underwent laparoscopic splenectomy two years back. Showing no response to splenectomy she was kept on dapsone therapy $100 \mathrm{mg}$ once daily. Past obstetrical history revealed spontaneous abortion at 18 weeks of gestational age. 
Laboratory test yielded the following results: haemoglobin $11.9 \mathrm{~g} / \mathrm{dl}(12-15 \mathrm{~g} / \mathrm{dl})$, total WBC count $11.4 \times 10^{9} / 1\left(4-10 \times 10^{9} / 1\right)$, hematocrit $39.5 \%(45-$ $50 \%)$, platelets $10 \times 10^{9} / 1\left(150-400 \times 10^{9} / 1\right)$, MCV 82.3 fl (83-101 fl), MCH 24.6 pg (27-31 pg), MCHC $29.8 \%(31.5-34.5 \%)$. PT/INR were within reference range. Blood borne virus screen for HIV, HbsAg, and $\mathrm{HCV}$ was negative.

Due to non-affordability, immunoglobulin or anti-D could not be given. The patient was started on high dose (1 gm intravenous infusion per day for 3 days) methylprednisolone (HDMP).The platelet counts increased to a level of $60,000 /$ cumm on third day after starting HDMP (Figure 1).

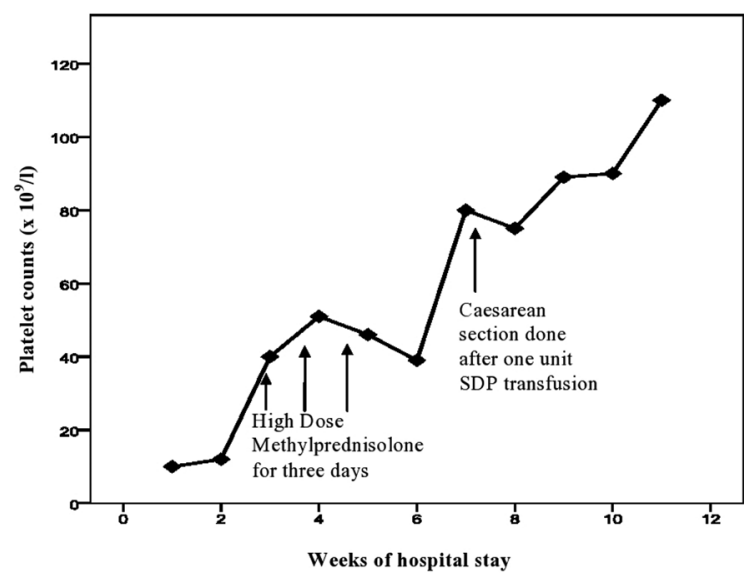

Figure 1. Platelet count of patient with treatment.

Thereafter patient was maintained on tapering dose of oral steroid for further three weeks and caesarean section was planned at 38 weeks with transfusion of single donor platelets (SDP) on the day of surgery. Male baby 3179 gm was delivered. Baby was also thrombocytopenic with platelet counts of $46 \times 10^{9} / 1$. Baby responded to immunoglobulin treatment with platelet counts increasing up to $78 \times 10^{9} / 1$. Mother and baby were discharged with platelet counts of $89 \mathrm{x}$ $10^{9} / 1$ and $91 \times 10^{9} / 1$ respectively.

\section{DISCUSSION}

Thrombocytopenia is the second most common hematological abnormality during pregnancy after anaemia. ${ }^{5}$ Thrombocytopenia during pregnancy due to physiological causes accounts for almost three fourths of all cases, and is often referred to as gestational thrombocytopenia. It may be secondary to accelerated platelet consumption and increased plasma volume associated with pregnancy. It is considered as a diagnosis of exclusion, and the following characteristics make gestational thrombocytopenia more likely: (1) the degree of thrombocytopenia is usually mild, usually remaining greater than $70,000 \mu \mathrm{L}$; (2) patients are asymptomatic with no history of bleeding and presenting usually in third trimester; (3) there is no preconception history of thrombocytopenia; (4) an early gestation or preconception platelet count is normal. ${ }^{6}$

Immune thrombocytopenic purpura incorporates pathological causes of thrombocytopenia in pregnancy, mainly comprising immune mediated platelet destruction. In acute form, it is usually selflimiting and mostly affecting children. When chronic, it predominately affects women in their second to third decades of life, and accounts for $3 \%$ of all cases of thrombocytopenia during pregnancy. ${ }^{7}$

The optimal treatment strategies for ITP in pregnancy still remain controversial. Therapy of the pregnant woman with ITP is very similar to that of nonpregnant individual. Therapeutic interventions are usually started when platelet counts reach unacceptably low values (approximately 30,000/uL), or when the patient exhibits signs of bleeding, such as petechiae, purpura, or epistaxis. ${ }^{8}$ Prednisolone (1 $\mathrm{mg} / \mathrm{kg}$ ) remains the first line of therapy for pregnant women with ITP, and the response to therapy is very much the same as that observed in non-pregnant individuals, with an overall response rate of $60 \%$ to $70 \% .{ }^{8}$ However, since steroid is not free from various side effects, IV immunoglobulins (IVIG) (1 gm/ $\mathrm{kg}$ once daily for 2 days) can be used alternatively which is considered as standard therapy by many experts for pregnancy associated thrombocytopenia due to its low toxicity profile. ${ }^{9}$ However, as IVIG due to its short lasting effect and high cost cannot be used in most of the patients. Intravenous anti-D, in a dose of 50-70 microgram $/ \mathrm{kg}$, having similar efficacy and toxicity profile as IVIG, and can also be used as second line therapy in patients not tolerating IVIG and/or steroid. ${ }^{10}$ Although it is cheaper than IVIG, it is still beyond the reach of most patients in India.

Use of HDMP (30 mg/kg for 3 days) as first line therapy in the management of ITP and also in patients not responding to conventional dose prednisolone (1 $\mathrm{mg} / \mathrm{kg} /$ day) has been well documented. HDMP has 
also been demonstrated to be effective in ITP patients refractory to IVIG and oral prednisolone. ${ }^{11}$ Narang et $\mathrm{a}^{12}$ have used HDPM successfully for managing acute crisis of thrombocytopenia, in third trimester of pregnancy of a non-splenectomized female suffering from ITP. Splenectomy which is considered as the standard treatment for ITP in adults has a response rate of $66 \% .{ }^{13}$ Strategies for treating a post-splenectomy refractory ITP still remains unexplored. We found HDMP to be effective in post-splenectomy refactory ITP. Hence we recommend using HDM in case of post-splenectomy refractory ITP during emergency situations.

ITP may affect both the mother and fetus, and the offspring of the patient with ITP may also develop thrombocytopenia, due to transplacental passage of maternal platelet autoantibodies. ${ }^{4}$ Hence active management of new born during first week of birth with IVIG should be considered.

\section{ACKNOWLEGEMENT}

We thank Dr Dipanwita Sen, department of obstetrics and gyaenecology of The Mission Hospital, Durgapur for her valuable support in providing relevant clinical details.

\section{DISCLOSURE}

The authors report no conflicts of interest in this work.

No violation of human rights and safety.

Funding: Nil

\section{REFERENCES}

1. Neloyn AJ, Saunders PW, Howard MR, Proctor SJ, Taylor RR. Clinically significant newly presenting autoimmune thrombocytopenic purpura in adults: a prospective study of population based cohort of 245 patients. Br J Haematol. 2003;122(6):966-74

2. Frederiksen H, Schmidt K. The incidence of idiopathic thrombocytopenic purpura in adult increases with age. Blood. 1999;94(3):909-13.

3. Kelton JG. Management of the pregnant patient with idiopathic thrombocytopenic purpura. Ann Intern Med. 1983;99(60):796-800.

4. Cines DB, Dusak B, Tomaski A, Mennuti M, Schreiber AD. Immune thrombocytopenic purpura and pregnancy. N Engl J Med. 1982;306(14):826-31

5. Sullivan CA, Martin JN. Management of the obstetric patient with thrombocytopenia. Clin Obstet Gynecol. $1995 ; 38(3): 521-34$
6. Silver R, Berkowitz R, Bussel J. Thrombocytopenia in pregnancy. Practice bulletin, No 6. Chicago: American College of Obstetrics and Gynecology; 1999.

7. Burrows RF, Kelton JG. Fetal thrombocytopenia and its relation to maternal thrombocytopenia. N Engl J Med. 1993;329(20):1463-6.

8. McCrae KR, Samuels P, Schreiber AD. Pregnancyassociated thrombocytopenia: pathogenesis and management. Blood. 1992;80(11):2697-714.

9. Gill KK, Kelton JG. Management of idiopathic thrombocytopenic purpura in pregnancy. Semin Hematol. 2000;37(30):275-89.

10. Michel M, Novoa MV, Bussel JB. Intravenous anti-D as a treatment for immune thrombocytopenic purpura (ITP) during pregnancy. Br J Haematol. 2003;123(1):142-6.

11. Godeau B, Zini JM, Schaeffer A, Bierling P. High dose methylprednisolone is an alternative treatment for adults with autoimmune thrombocytopenic purpura refractory to intravenous immunoglobulins and oral corticosteroids. Am J Hematol. 1995;48(4):282-4.

12. Narang M, Sunita, Bhasin S, Saluja S, Sharma M, Gupta $\mathrm{DK}$, et al. Idiopathic thrombocytopenic purpura in pregnancy: a therapeutic challenge. J Ind Acad Clin Med 2006;7(2):147-49

13. Kojouri K, Vesely SK, Terrell DR, George JN. Splenectomy for adult patients with idiopathic thrombocytopenic purpura: a systematic review to assess long-term platelet count responses, prediction of response, and surgical complications. Blood. 2004;104(9):2623-34. 